\title{
NOTE ON THE DERIVATIVES OF FUNCTIONS ANALYTIC IN THE UNIT CIRCLE
}

\author{
J. L. WALSH
}

In a recent paper, ${ }^{1} \mathrm{~W}$. Seidel and the present writer established distortion theorems for various classes of functions analytic in the unit circle; more explicitly, established relations between the derivatives of functions and their radii of univalence and of $p$-valence, with particular reference to behavior as a point approaches the circumference. Classes studied in detail were functions respectively univalent, bounded, omitting two values, $p$-valent, and having a bounded radius of univalence. The last-named class clearly includes the class of functions $f(z)$ each analytic in $|z|<1$ and transforming $|z|<1$ onto a Riemann configuration of finite area. It is the primary object of the present note to study this included class to the same end more effectively by other methods. Terminology and notation are uniform with the paper referred to; unless otherwise specified, references in the present note are to that paper; we shall also have occasion to use the results of a subsequent paper by Loomis. ${ }^{2}$

We denote by $K_{M}$ the class of functions $f(z)$ analytic in $|z|<1$ and transforming the region $|z|<1$ into a region whose area (counted according to the multiplicity of covering) is not greater than $\pi M^{2: 3}$

$$
\iint_{|z|<1}\left|f^{\prime}(z)\right|^{2} d S_{\mathrm{s}} \leqq \pi M^{2}
$$

If we set $f(z) \equiv \sum_{0}^{\infty} a_{n} z^{n}$, inequality (1) becomes

$$
\sum_{1}^{\infty} n\left|a_{n}\right|^{2} \leqq M^{2}
$$

whence we have

$$
\left|a_{n}\right| \leqq \frac{M}{n^{1 / 2}} .
$$

Received by the editors November 5, 1946.

1 Trans. Amer. Math. Soc. vol. 52 (1942) pp. 128-216.

2 Bull. Amer. Math. Soc. vol. 48 (1942) pp. 908-911.

${ }^{3}$ Added in proof. This class of functions has recently been studied by P. Montel (Publicaciones del Instituto de Matemática de la Universidad Nacional del Litoral, Rosario, vol. 6 (1946) pp. 273-286), who obtains sharp inequalities improving (12) and (13) below, but does not prove any of the italicized theorems of the present note. Compare also T. H. Gronwall, Ann. of Math. vol. 16 (1914) pp. 72-76. 
We proceed now to establish the following theorem.

THEOREM 1. If $f(z)$ is of class $K_{M}$, with $w_{0}=f\left(z_{0}\right)$, we have

$$
D_{1}\left(w_{0}\right) \leqq\left|f^{\prime}\left(z_{0}\right)\right|\left(1-\left|z_{0}\right|^{2}\right) \leqq\left[6 M D_{1}\left(w_{0}\right)\right]^{1 / 2} .
$$

The first inequality in (4) has already been established (loc. cit. \$4). We proceed to prove the second inequality in (4).

The proof is by the method of Landau employed in the previous paper (\$18). Let us assume first $f(0)=0, f^{\prime}(0)=1$, from which it follows by (3) that the series $\sum a_{n} z^{n}$ is term by term not greater in absolute value than the series

$$
z+M z^{2}+M z^{3}+M z^{4}+\cdots ;
$$

the latter series has the sum $(|z|<1)$

$$
z+\frac{M z^{2}}{1-z}
$$

Of course we have $M \geqq 1$.

For the value $r=1 /(4 M)$ we have

(7) $r-\max _{|z|=r}|f(z)-z| \geqq r-\frac{M r^{2}}{1-r} \geqq r-\frac{4 M r^{2}}{3}=\frac{1}{6 M}=\phi(M)$.

Thus on the circle $|z|=r$ we have

$$
\left|\frac{f(z)-z}{z-w}\right|<1
$$

for $|w|<\phi(M)$, so by Rouchés theorem $f(z)$ takes on the value $w$ precisely as many times in $|z|<r$ as does the function $z$, namely once. That is to say, the map $w=f(z),|z|<1 /(4 M)$, covers smoothly the circle $|w|<\phi(M)=1 /(6 M)$. Thus we have

$$
D_{1}(0) \geqq 1 / 6 M \text {. }
$$

Given an arbitrary $z_{0}$, with $\left|z_{0}\right|<1$, the case $f^{\prime}\left(z_{0}\right)=0$ trivially implies (4), with each member zero; in the case $f^{\prime}\left(z_{0}\right) \neq 0$ we construct the frequently used auxiliary function

$$
\psi(z)=\frac{f\left(\left(z+z_{0}\right) /\left(1+\bar{z}_{0} z\right)\right)-f\left(z_{0}\right)}{f^{\prime}\left(z_{0}\right)\left(1-\left|z_{0}\right|^{2}\right)} .
$$

We have $\psi(0)=0, \quad \psi^{\prime}(0)=1$. Since the transformation $z^{\prime}$ $=\left(z+z_{0}\right) /\left(1+\bar{z}_{0} z\right)$ maps $|z|<1$ smoothly onto $\left|z^{\prime}\right|<1$, it is clear that the Riemann configuration which is the image of $|z|<1$ under 
the transformation $w=f\left(z^{\prime}\right)$ is the same as the image of $|z|<1$ under the transformation $w=f(z)$; consequently $\psi(z)$ is of class $K_{M^{\prime}}$, with

$$
M^{\prime}=\frac{M}{\left|f^{\prime}\left(z_{0}\right)\right|\left(1-\left|z_{0}\right|^{2}\right)} .
$$

By inequality (8) we may now write

$$
D_{1}\left(w_{0}\right) \geqq \frac{\left|f^{\prime}\left(z_{0}\right)\right|\left(1-\left|z_{0}\right|^{2}\right)}{6 M},
$$

where $D_{1}\left(w_{0}\right)$ here refers to the image of $|z|<1$ under the transformation $w=\psi(z)$. By equation (9) we obviously have

$$
\left.D_{1}\left(w_{0}\right)\right|_{w=\psi(z)}=\left.D_{1}\left(w_{0}\right)\right|_{w=f(z)} \div\left|f^{\prime}\left(z_{0}\right)\right|\left(1-\left|z_{0}\right|^{2}\right)
$$

which implies the second inequality in (4) and completes the proof of Theorem 1.

The order $1 / 2$ as $D_{1}\left(w_{0}\right)$ in (4) approaches zero cannot be improved; compare loc. cit. $\$ 10$.

This method just used yields a slightly more favorable inequality than (4). Series (5) can be replaced by

$$
z+\frac{M z^{2}}{2^{1 / 2}}+\frac{M z^{3}}{2^{1 / 2}}+\frac{M z^{4}}{2^{1 / 2}}+\cdots=z+\frac{M z^{2}}{2^{1 / 2}(1-z)} .
$$

Then the latter part of (7) can be replaced by

$$
r-\frac{M r^{2}}{2^{1 / 2}(1-r)} \geqq r-\frac{4 M r^{2}}{3 \cdot 2^{1 / 2}}=\frac{6-2^{1 / 2}}{24 M}=\phi(M) .
$$

The second member of ( 8$)$ is $\phi(M)$, so in (4) the number 6 may be replaced by

$$
\frac{24}{6-2^{1 / 2}}=\frac{72+12 \cdot 2^{1 / 2}}{17}=5.24
$$

We remark too that in (10) the number $M$ can be replaced by $\left(M^{2}-1\right)^{1 / 2}$ with a slight improvement in (4).

In extending Theorem 1 to higher derivatives, we shall prove the following theorem.

THEOREM 2. If the function $f(z)=\sum_{0}^{\infty} a_{n} z^{n}$ is of class $K_{M}$, then we have

$$
\begin{aligned}
D_{p}(0) & \leqq \sum_{1}^{p}\left|a_{n}\right| \leqq 2^{p} \cdot A_{p}(M \log 2)^{p /(p+1)}\left[D_{p}(0)\right]^{1 /(p+1)} \\
A_{p} & =(p+1)[(p+1) / p]^{p /(p+1)}(4 p)^{p}
\end{aligned}
$$


The first inequality in (11) is established by Loomis (loc. cit.), a sharpening of an inequality due to Seidel and Walsh. To establish the second inequality we write for $\left|z_{0}\right|<1$, with the double integral taken over $\left|z-z_{0}\right|<1-\left|z_{0}\right|$,

$$
\left|f^{\prime}\left(z_{0}\right)\right|^{2}=\frac{1}{\pi\left(1-\left|z_{0}\right|\right)^{2}}\left|\iint\left[f^{\prime}(z)\right]^{2} d S\right| \leqq \frac{M^{2}}{\left(1-\left|z_{0}\right|\right)^{2}} .
$$

If we assume $f(0)=0$, which here involves no loss of generality, we have

$$
\begin{array}{r}
|f(z)|=\left|\int_{0}^{z} f^{\prime}(t) d t\right| \leqq \int_{0}^{r} \frac{M d t}{1-t}=-M \log (1-r), \\
|z|=r<1 .
\end{array}
$$

The function $F(z) \equiv f(z / 2) \equiv a_{1}(z / 2)+a_{2}(z / 2)^{2}+\cdots$ is analytic in the circle $|z|<1$ and by (13) is of modulus not greater than $M \log 2$ there. Consequently we have (Loomis, loc. cit.)

$$
\frac{\left|a_{1}\right|}{2}+\frac{\left|a_{2}\right|}{2^{2}}+\cdots+\frac{\left|a_{p}\right|}{2^{p}} \leqq A_{p}(M \log 2)^{p /(p+1)}\left[D_{p}(0)\right]^{1 /(p+1)}
$$

where $D_{p}(0)$ refers to the largest $m$-sheeted circle $(m \leqq p)$ with center $O$ and contained in the image of $|z|<1$ under the transformation $w=F(z)$. Inequality (14), valid where $D_{p}(0)$ refers to the image of $|z|<1 / 2$ under the transformation $w=f(z)$, is a fortiori valid if $D_{p}(0)$ refers to the image of $|z|<1$ under the transformation $w=f(z)$, and (11) follows at once.

If inequality (11) is rewritten so as to apply to the function

$$
g(\zeta)=f\left(\frac{\zeta+z}{1+\zeta \bar{z}}\right), \quad|z|<1,|\zeta|<1,
$$

it appears (Siedel and Walsh, loc. cit. Chapter I, Lemma 2) that $D_{p}\left(w_{n}\right) \rightarrow 0$ is a necessary and sufficient condition for the relations

$$
f^{(m)}\left(z_{n}\right)\left(1-\left|z_{n}\right|^{2}\right)^{m} \rightarrow 0, \quad m=1,2, \cdots, p .
$$

For functions $f(z)$ of class $K_{M}$, this remark establishes the relation $D_{p}\left(w_{n}\right) \rightarrow 0$ as a consequence of $w_{n}=f\left(z_{n}\right),\left|z_{n}\right| \rightarrow 1$, by virtue of the following theorem:

THEOREM 3. If $f(z)$ belongs to $K_{M}$, then we have for every $k$

$$
\lim _{|z| \rightarrow 1} f^{(k)}(z)\left(1-|z|^{2}\right)^{k}=0 ;
$$


the approach is uniform in the sense that if $\epsilon>0$ is given, there exists $\delta_{\epsilon}$ such that $1-|z|<\delta_{\text {, }}$ implies $\left|f^{(k)}(z)\left(1-|z|^{2}\right)^{k}\right|<\epsilon$.

Of course the uniformity of the approach is a consequence of the existence of the limit. We introduce the notation

$$
\iint_{r<|z|<1}\left|f^{\prime}(z)\right|^{2} d S=\Phi(r),
$$

whence $\lim _{r \rightarrow 1} \Phi(r)=0$ monotonically. If $\rho(>1 / 2)$ is arbitrary, $\rho<\left|z_{0}\right|$ $<1$, we choose $r \leqq 2 \rho-1$, and the integral in (12) is in absolute value not greater than $\Phi(r)$, so we have

$$
\left|f^{\prime}\left(z_{0}\right)\right|^{2} \leqq \frac{\Phi(r)}{\pi\left(1-\left|z_{0}\right|\right)^{2}},
$$

from which (15) follows uniformly, for the value $k=1$.

We now take $|z|>1 / 3, \rho=(1-|z|) / 2$, and use Cauchy's integral formula

$$
f^{\prime}(z)=\frac{1}{2 \pi i} \int_{|t-z|=\rho} \frac{f^{\prime}(t) d t}{t-z} .
$$

Successive differentiation yields

$$
f^{(k)}(z)=\frac{(k-1) !}{2 \pi i} \int_{|t-z|=\rho} \frac{f^{\prime}(t) d t}{(t-z)^{k}},
$$

whence we have

$$
\left|f^{(k)}(z)\right| \leqq(k-1) !\left[\max \left|f^{\prime}(t)\right|, \text { for }|t-z|=\rho\right] / \rho^{k-1},
$$

and by the definition of $\rho$,

$(1-|z|)^{k-1}\left|f^{(k)}(z)\right| \leqq 2^{k-1} \cdot(k-1) !\left[\max \left|f^{\prime}(t)\right|\right.$, for $\left.|t-z|=\rho\right]$.

We have further $(1-|t|) \geqq(1-|z|) / 2$, so we deduce

$$
\begin{aligned}
& (1-|z|)^{k}\left|f^{(k)}(z)\right| \\
& \quad \leqq 2^{k-1}(k-1) !(1-|z|)\left[\max \left|f^{\prime}(t)\right|, \text { for }|t-z|=\rho\right] \\
& \quad \leqq 2^{k}(k-1) !\left[\max \left|f^{\prime}(t)\right|(1-|t|), \text { for }|t-z|=\rho\right],
\end{aligned}
$$

which implies (15) uniformly and establishes Theorem 3.

It may be noted that we have derived (15) for $k>1$ merely from (15) for $k=1$ as a consequence only of the analyticity of $f(z)$ in $|z|<1$.

For the class $K_{M}$ we can make no deductions regarding the rapidity of approach to zero in (15): 
THEOREM 4. Let the function $Q(r)$ be defined and positive for $0<r<1$, with $\lim _{r \rightarrow 1} Q(r)=0$. Let the positive integer $m$ and the positive number $M$ be given. Then there exists a function $f(z)$ of class $K_{M}$, continuous and schlicht for $|z| \leqq 1$, and there exists a sequence of points $z_{k}$ with $0<z_{k}<1$, $z_{k} \rightarrow 1$, such that we have

$$
\lim _{k \rightarrow \infty} \frac{f^{(m)}\left(z_{k}\right)\left(1-\left|z_{k}\right|^{2}\right)^{m}}{Q\left(\left|z_{k}\right|\right)}=\infty .
$$

It suffices to choose here a suitable constant multiple of the function $f(z)$ previously constructed (loc. cit. §9).

It is clear from Theorem 1 that the two conditions

$$
\begin{aligned}
D_{1}\left(w_{n}\right) \rightarrow 0, \quad w_{n} & =f\left(z_{n}\right), \\
f^{\prime}\left(z_{n}\right)\left(1-\left|z_{n}\right|^{2}\right) & \rightarrow 0,
\end{aligned}
$$

for a function $f(z)$ of class $K_{M}$ are equivalent in the sense that each implies the other. If $z_{n}$ is a sequence $\left(\left|z_{n}\right|<1\right)$ which has no limit point in a zero of $f^{\prime}(z)$ interior to $|z|=1$, it is clear that (18) implies

$$
\left|z_{n}\right| \rightarrow 1 \text {; }
$$

reciprocally (19) implies (18) by virtue of Theorem 3 . That is to say, for a function of class $K_{M}$ these three conditions are all equivalent, except that (17) and (18) are trivially fulfilled for any sequence or subsequence $z_{n}$ which approaches a zero of $f^{\prime}(z)$ interior to $|z|=1.4$

Theorem 3 asserts that if $f(z)$ belongs to $K_{M}$, then (19) implies (15) for every $k$. We have remarked that Theorem 2 as applied to the function

shows that

$$
f\left(\frac{\zeta+z}{1+\bar{z} \zeta}\right)
$$

$$
\lim _{n \rightarrow \infty} D_{p}\left(w_{n}\right)=0
$$

is equivalent to (15) for $k=1,2, \cdots, p$. Thus for a function $f(z)$ of class $K_{M}$, the three relations (19), (20), and (15) for $k=1,2, \cdots, p$ are all equivalent except that the latter two relations are trivially fulfilled for any sequence or subsequence $z_{n}$ which approaches a common zero of $f^{(k)}(z), k=1,2, \cdots, p$, interior to $|z|=1$.

\footnotetext{
4 In the study of the equivalence of the relations (17), (18), and (19) for bounded univalent functions (loc. cit. p. 211), the phrase "every point of $R_{N_{\delta}}$ " should read "every point of $R$ exterior to $R_{N_{\delta}}$."
} 
In considering the relationships between (17), (18), and (19), it is of interest to study the geometric significance of (17) independently of the class $K_{M}$. We shall prove:

THEOREM 5. Let the function $w=f(z)$ be analytic interior to $|z|<1$, and map $|z|<1$ onto the Riemann configuration $R$. A necessary and sufficient condition that (19) imply (17) with $w_{n}=f\left(z_{n}\right)$ is that there exist no $\delta(>0)$ to which correspond an infinity of mutually nonoverlapping smooth circles $C_{1}, C_{2}, \cdots$ of radius $\delta$ in $R$.

If for some $\delta(>0)$ such circles $C_{n}$ do exist, their respective centers $w_{n}$ (considered as points of $R$, not merely as values of the complex variable) have no limit point in $R$, and the corresponding points $z_{n}$ have no limit point interior to $|z|<1$; thus (19) is satisfied but not (17), so (19) does not imply (17). Conversely, let us assume that (19) does not imply (17); we suppose (19) satisfied for a particular sequence $z_{n}$, but suppose that (17) is not satisfied; we shall show the existence of a suitable $\delta(>0)$ and corresponding circles $C_{n}$ in $R$. Choose a subsequence of the $z_{n}$ so that for a suitably chosen $\delta(>0)$ the values $D_{1}\left(w_{n}\right)$ are all greater than or equal to $2 \delta$; we change the notation if necessary so as to have $D_{1}\left(w_{n}\right) \geqq 2 \delta$ for every $n$. If any subsequence $\Gamma_{n_{k}}$ of these circles $\Gamma_{n}$ (with respective centers $w_{n}$ and radii $D_{1}\left(w_{n}\right)$ ) have centers $w_{n_{k}}$ in $R$ which approach as limit a point $w_{0}$ of $R$, then (loc. cit. $\left.\S 15\right)$ by the continuity of $D_{1}(w)$ as a function of $w$, we have $D_{1}\left(w_{0}\right) \geqq 2 \delta$; consequently we have $w_{n_{k}} \rightarrow w_{0}, z_{n_{k}} \rightarrow z_{0}$ with $\left|z_{0}\right|<1$, contrary to hypothesis. At most a finite number of the points $w_{2}, w_{3}, \cdots$ lie interior to the circle $\gamma_{1}$ whose center is $w_{1}$ and radius $2 \delta$; suppose none of the points $w_{n}$ for $n \geqq N_{1}$ lies interior to $\gamma_{1}$. At most a finite number of the points $w_{n}$ for $n>N_{1}$ lie interior to the circle $\gamma_{2}$ whose center is $w_{N_{1}}$ and radius $2 \delta$; suppose none of the points $w_{n}$ for $n \geqq N_{2}\left(>N_{1}\right)$ lies interior to $\gamma_{2}$. Let $\gamma_{3}$ be the circle whose center is $w_{N_{2}}$ and radius $2 \delta$. We continue this process indefinitely, and find a sequence of circles $\gamma_{1}, \gamma_{2}, \cdots$ all of radius $2 \delta$; the center of $\gamma_{k}$ cannot lie interior to any of the circles $\gamma_{1}, \gamma_{2}, \cdots, \gamma_{k-1}$. The circles $C_{k}$ concentric with the circles $\gamma_{k}$ and having the common radius $\delta$ lie in $R$ and no two of them overlap. The proof is complete.

The condition of Theorem 5 is equivalent to the condition that there exist no $\epsilon(>0)$ such that for every $n=1,2,3, \cdots$, the image $R_{n}$ of the annulus $1>|z|>1-1 / n$ under the transformation $w=f(z)$ contains a smooth circle of radius $\epsilon$. Let each $R_{n}$ contain a smooth circle $\Gamma_{n}$ of radius $\epsilon$; then $R_{n}$ contains the concentric circle $C_{n}$ of radius $\delta=\epsilon / 2$. Points of each $C_{n}$ lie in but a finite number of regions $R_{k}$, so there exist among the $C_{n}$ an infinite number of nonoverlapping 
smooth circles in $R$, each of radius $\delta$. Conversely, let there exist an infinity of nonoverlapping smooth circles $\Gamma_{n}$ of radius $\delta(>0)$ in $R$; let $C_{n}$ denote the concentric circles of radius $\epsilon=\delta / 2$, and $w_{n}$ denote the respective centers. Any closed region $R-R_{k}$ contains points belonging to at most a finite number of the circles $C_{n}$, for otherwise there exists a point $z_{0}$ with $\left|z_{0}\right| \leqq 1-1 / k$ such that every neighborhood of $z_{0}$ contains points $z$ corresponding to points $w$ of an infinity of those circles $C_{n}$; every neighborhood in $R$ of the point $w_{0}=f\left(z_{0}\right)$ then contains points of an infinity of the circles $C_{n}$, which is impossible. Consequently each $R_{k}$ contains at least one $C_{n}$; the equivalence is established.

As a consequence of Theorem 5 we prove:

COROLLARY 1. Let the function $w=f(z)$ be analytic interior to $|z|<1$, and map $|z|<1$ onto the Riemann configuration $R$. A necessary and sufficient condition that (19) imply (20) for each $p$ is that there exist no $\delta(>0)$ to which correspond an infinity of mutually nonoverlapping smooth circles of radius $\delta$ in $R$.

We have the inequality

$$
D_{1}(w) \leqq D_{p}(w),
$$

so if (19) implies (20) for a single value of $p$, then (19) also implies $(17)$, and by Theorem 5 there exists no $\delta(>0)$ to which correspond an infinity of mutually nonoverlapping smooth circles of radius $\delta$ in $R$. Conversely, for given $p$ suppose (19) does not imply (20); we assume (19) satisfied for a particular sequence $z_{n}$ but assume (20) for a particular $p$ not satisfied; we shall show the existence of a suitable $\delta$ and the corresponding circles. By the method of proof of Theorem 5 there exists a $\delta(>0)$ and a sequence of $m$-sheeted circles $(m \leqq p)$ $\Gamma_{1}, \Gamma_{2}, \cdots$, of radius not less than $2 \delta$, which belong to $R$ and are mutually nonoverlapping. Each circle $\Gamma_{j}$ contains at least one smooth circle $C_{j}$ of radius $\delta ;$ for an $m$-sheeted circle has branch points of total multiplicity $m-1$; a branch point of order $m-1$ lies in $m$ sheets, a branch point of order $m-2$ lies in $m-1$ sheets, $\cdots$, a branch point of order 1 lies in 2 sheets; at least two sheets of $\Gamma_{j}$ contain but one branch point; and each of these two sheets contains a smooth circle of radius $\delta$. The circles $C_{j}$ are mutually nonoverlapping, so Corollary 1 is established. If for any function analytic in $|z|<1$ condition (19) implies (17), then (19) also implies (20) for every $p$.

The condition of Theorem 5 is obviously satisfied by any function

5 This fact follows also from a lemma due to Grünwald and Turán as sharpened by Grünwald and Vázsonyi; see Acta Univ. Szeged. vol. 8 (1936-1937) pp. 236-240. 
of class $K_{M}$, and by functions bounded or unbounded of many other types. Moreover we prove the following corollary.

CoROllary 2. If $f(z)$ is analytic and schlicht in $|z|<1$, and maps $|z|<1$ onto a region $R$, then a necessary and sufficient condition for the equivalence of (17), (18), and (19) is that there exist no $\delta(>0)$ to which correspond an infinity of mutually nonoverlapping smooth circles $C_{n}$ of radius $\delta$ in $R$.

We have (loc. cit. \$4) the inequalities

$$
D_{1}\left(w_{0}\right) \leqq\left|f^{\prime}\left(z_{0}\right)\right|\left(1-\left|z_{0}\right|^{2}\right) \leqq 4 D_{1}\left(w_{0}\right),
$$

from which the equivalence of (17) and (18) follows. The derivative $f^{\prime}(z)$ is different from zero at all points in $|z|<1$, so (18) must imply (19). Corollary 2 now follows from Theorem 5.

For an arbitrary function $f(z)$ analytic in $|z|<1$, the existence of $\delta(>0)$ and corresponding mutually nonoverlapping smooth circles $C_{j}$ in $R$ of radius $\delta$ implies

$$
\limsup _{|z| \rightarrow 1}\left|f^{\prime}(z)\right|\left(1-|z|^{2}\right)>0
$$

by the first of inequalities (21); see loc. cit. $\$ 4$.

HARVARD UNIVERSITY 